UT-08-10

\title{
M5 from M2
}

\author{
Pei-Ming $\mathrm{Ho}^{\dagger 1}$, Yutaka Matsuo ${ }^{\ddagger 2}$ \\ $\dagger$ Department of Physics and Center for Theoretical Sciences, \\ National Taiwan University, Taipei 1061\%, Taiwan, R.O.C. \\ $\ddagger$ Department of Physics, Faculty of Science, University of Tokyo, \\ Hongo 7-3-1, Bunkyo-ku, Tokyo 113-0033, Japan
}

\begin{abstract}
Recently an action based on Lie 3-algebras was proposed to describe M2-branes. We study the case of infinite dimensional Lie 3-algebras based on the Nambu-Poisson structure of three dimensional manifolds. We show that the model contains self-dual 2-form gauge fields in 6 dimensions, and the result may be interpreted as the M5-brane world-volume action.
\end{abstract}

\footnotetext{
${ }^{1}$ e-mail address: pmho@phys.ntu.edu.tw

${ }^{2}$ e-mail address: matsuo@phys.s.u-tokyo.ac.jp
} 


\section{Introduction}

Recently, Bagger and Lambert [1-3] and Gustavsson [4,5] proposed a supersymmetric Lagrangian for multiple M2-branes with a Lie 3-algebra [6] as the internal symmetry

$$
\mathcal{L}=-\frac{1}{2}\left\langle D^{\mu} X^{I}, D_{\mu} X^{I}\right\rangle+\frac{i}{2}\left\langle\bar{\Psi}, \Gamma^{\mu} D_{\mu} \Psi\right\rangle+\frac{i}{4}\left\langle\bar{\Psi}, \Gamma_{I J}\left[X^{I}, X^{J}, \Psi\right]\right\rangle-V(X)+\mathcal{L}_{C S},
$$

where $D_{\mu}$ is the covariant derivative

$$
\left(D_{\mu} X^{I}(x)\right)_{a}=\partial_{\mu} X_{a}^{I}-f^{c d b}{ }_{a} A_{\mu c d}(x) X_{b}^{I},
$$

$V(X)$ is the potential term defined by

$$
V(X)=\frac{1}{12}\left\langle\left[X^{I}, X^{J}, X^{K}\right],\left[X^{I}, X^{J}, X^{K}\right]\right\rangle,
$$

and the Chern-Simons action for the gauge potential is

$$
\mathcal{L}_{C S}=\frac{1}{2} \epsilon^{\mu \nu \lambda}\left(f^{a b c d} A_{\mu a b} \partial_{\nu} A_{\lambda c d}+\frac{2}{3} f_{g}^{c d a} f^{e f g b} A_{\mu a b} A_{\nu c d} A_{\lambda e f}\right) .
$$

The indices $I, J, K$ run in $1, \cdots, 8$, and they specify the transverse directions of $M 2$-brane; $\mu, \nu$ run in $0,1,2$, describing the longitudinal directions. The indices $a, b, c$ take values in $1, \cdots, \mathcal{D}$ where $\mathcal{D}$ is the number of generators of the Lie 3-algebra specified by a set of structure constants $f^{a b c}{ }_{d}$.

The fermionic field $\Psi$ is a Majorana spinor in $10+1$ dimensions satisfying the chirality condition $\Gamma_{012} \Psi=-\Psi$. (The SUSY parameter $\epsilon$ satisfies $\Gamma_{012} \epsilon=\epsilon$.) As a result $\Psi$ has 16 real fermionic components, equivalent to 8 bosonic degrees of freedom. The bosonic fields include $8 X^{I}$ 's and $3 A_{\mu}$ 's. In $2+1$ dimensions, ordinarily a gauge potential has one propagating degree of freedom. However, here the gauge potential $A$ has no canonical kinetic term, but only a Chern-Simons term, and hence it has no propagating degree of freedom.

The action has $N=8$ maximal SUSY in $d=3$, and the SUSY transformations are

$$
\begin{aligned}
\delta X_{a}^{I} & =i \bar{\epsilon} \Gamma^{I} \Psi_{a}, \\
\delta \Psi_{a} & =D_{\mu} X_{a}^{I} \Gamma^{\mu} \Gamma^{I} \epsilon-\frac{1}{6} X_{b}^{I} X_{c}^{J} X_{d}^{K} f^{b c d}{ }_{a} \Gamma^{I J K} \epsilon, \\
\delta \tilde{A}_{\mu}{ }^{b}{ }_{a} & =i \bar{\epsilon} \Gamma_{\mu} \Gamma_{I} X_{c}^{I} \Psi_{d} f^{c d b}{ }_{a} .
\end{aligned}
$$

The gauge symmetry for the bosonic fields are written as,

$$
\delta X_{a}^{I}=\Lambda_{c d} f_{a}^{c d b} X_{b}^{I}, \quad \delta \tilde{A}_{\mu}{ }^{b}{ }_{a}=\partial_{\mu} \tilde{\Lambda}_{a}^{b}-\tilde{\Lambda}_{c}^{b} \tilde{A}_{\mu}{ }^{c}{ }_{a}+\tilde{A}_{\mu}{ }^{b}{ }_{c} \tilde{\Lambda}_{a}^{c} .
$$

For the consistency of these symmetries, we need to require a generalized Jacobi identity (or the fundamental identity) to the structure constants,

$$
\sum_{g} f_{c d e}{ }^{g} f_{a b g}{ }^{h}=\sum_{g}\left(f_{a b c}{ }^{g} f_{g d e}{ }^{h}+f_{a b d}{ }^{g} f_{c g e}{ }^{h}+f_{a b e}{ }^{g} f_{c d g}{ }^{h}\right) .
$$

When $\mathcal{D}$ is finite and the metric $h^{a b}=\left\langle T^{a}, T^{b}\right\rangle$ for the basis of generators $T^{a}(a=1, \cdots, \mathcal{D})$ is positive definite, the only known examples are (1) trivial algebra $f^{a b c}{ }_{d}=0,(2)$ the so-called $\mathcal{A}_{4}$ [7], with $\mathcal{D}=4$ and the structure constant $f_{d}^{a b c}=i \epsilon_{a b c d}$, and (3) their direct sums. Many attempts have been made 
to search for further nontrivial examples of 3-algebras [8-11]. It was conjectured in [10] that there exists no other example of finite dimension with a positive definite metric. This was finally proved in $[11,12]$ very recently.

When the constraints (1) $\mathcal{D}$ is finite and/or (2) $h^{a b}$ is positive definite are replaced by milder constraints, there are many varieties of 3 -algebras which satisfy the fundamental identity (see for example $[10,13])$. In particular in [10], we commented that for any manifolds with Nambu-Poisson structure [14-18], one can define $\mathcal{D}=\infty$ positive-definite Lie 3 -algebra.

The BLG theory has gained a lot of attention very recently [19]. In this paper, we examine the BLG theory with $\mathcal{D}=\infty$ Lie 3 -algebras based on 3 manifolds $\mathcal{N}$ with Nambu-Poisson structures. We will show that the field theory on the membrane world volume $\mathcal{M}$ can be rewritten as field theory on a 6 dimensional manifold $\mathcal{M} \times \mathcal{N}$ whose bosonic components consist of the self-dual gauge field on $\mathcal{M} \times \mathcal{N}$ and scalar fields which define the embedding. As this is the field content of an M5 brane [20,21], we interpret it as a model of M5-brane constructed out of infinitely many M2-branes. We note that this problem was considered in Basu and Harvey [22] in the context of the generalized Nahm equation. Our result will provide a new prospective to this problem.

\section{Nambu-Poisson manifold and Lie 3-algebra}

We consider 3 dimensional manifolds $\mathcal{N}$ equipped with the Nambu-Poisson brackets. The NambuPoisson bracket is a multi-linear map from $C(\mathcal{N})^{\otimes 3}$ to $C(\mathcal{N})$ defined as

$$
\left\{f_{1}, f_{2}, f_{3}\right\}=\sum_{\dot{\mu}, \dot{\nu}, \dot{\lambda}} P_{\dot{\mu} \dot{\nu} \dot{\lambda}}(y) \partial_{\dot{\mu}} f_{1} \partial_{\dot{\nu}} f_{2} \partial_{\dot{\lambda}} f_{3},
$$

where $P_{\dot{\mu}, \dot{\nu}, \dot{\lambda}}$ is an anti-symmetric tensor. We use the coordinate $y^{\dot{\mu}}(\dot{\mu}=1,2,3)$ to parametrize $\mathcal{N}$. The Nambu-Poisson bracket needs to satisfy the fundamental identity,

$$
\left\{g, h,\left\{f_{1}, f_{2}, f_{3}\right\}\right\}=\left\{\left\{g, h, f_{1}\right\}, f_{2}, f_{3}\right\}+\left\{f_{1},\left\{g, h, f_{2}\right\}, f_{3}\right\}+\left\{f_{1}, f_{2},\left\{g, h, f_{3}\right\}\right\} .
$$

which gives severe constraints on $P_{\dot{\mu}, \dot{\nu}, \dot{\lambda}}(y)$ (see for example [18]).

The simplest possible Nambu-Poisson bracket is the Jacobian determinant for 3 variables $y_{\dot{\mu}}(\dot{\mu}=$ $1,2,3)$

$$
\left\{f_{1}, f_{2}, f_{3}\right\}=\sum_{\dot{\mu}, \dot{\nu}, \dot{\lambda}} \epsilon_{\dot{\mu} \dot{\nu} \dot{\lambda}} \partial_{\dot{\mu}} f_{1} \partial_{\dot{\nu}} f_{2} \partial_{\dot{\lambda}} f_{3} .
$$

This is the classical Nambu bracket. In general, it is known that a consistent Nambu bracket reduces to this Jacobian form locally by the change of local coordinates [17]. So we will use it in the following for simplicity.

Nambu-Poisson bracket may be regarded as the definition of Lie 3-algebra in the infinite dimensional space $C(\mathcal{N})$. We write the basis of $C(\mathcal{N})$ as $\chi^{a}(y)(a=1,2, \cdots, \infty)$. We define the Lie 3-algebra structure constant by Nambu-Poisson bracket

$$
\left\{\chi^{a}, \chi^{b}, \chi^{c}\right\}:=\sum_{\dot{\mu} \dot{\nu} \dot{\lambda}} \epsilon_{\dot{\mu} \dot{\nu} \dot{\lambda}} \partial_{\dot{\mu}} \chi^{a} \partial_{\dot{\nu}} \chi^{b} \partial_{\dot{\lambda}} \chi^{c}=\sum_{d} f_{d}^{a b c} \chi^{d}(y) .
$$

We write the inner product as integration

$$
(\chi, \phi)=\int_{\mathcal{N}} d^{p} y \mu(y) \chi(y) \phi(y) .
$$


The measure factor $\mu$ is chosen such that the inner product is invariant under the Nambu-Poisson bracket, namely

$$
\left(\left\{f_{1}, f_{2}, \chi\right\}, \phi\right)+\left(\chi,\left\{f_{1}, f_{2}, \phi\right\}\right)=0 .
$$

The inner product between the basis elements $h^{a b}=\left(\chi^{a}, \chi^{b}\right)$ is called metric. We choose the dual set of basis $\chi_{a}(Y)$ in $C(\mathcal{N})$ such that $\left(\chi_{a}, \chi^{b}\right)=\delta_{b}^{a}$. We write $\left(\chi_{a}, \chi_{b}\right)=h_{a b}$ and $\sum_{b} h^{a b} h_{b c}=\delta_{c}^{a}$. The indices of the structure constant can be changed by contraction of the metric. In particular, $\sum_{e} h^{a e} f_{e}^{b c d}=f^{b c d a}$ defines a totally anti-symmetric 4-tensor. In order to have finite metric, we need to restrict $\mathcal{N}$ to a compact manifold. One may, of course, discuss noncompact manifolds by appropriate limits of the compact spaces.

\subsection{Examples}

Since we will analyze only the quadratic terms of the Lagrangian, the detail the 3-algebra will not be so relevant. However, since it is of some interest to see the algebra itself explicitly, we present a few examples where explicit computation is possible. It will be useful to proceed to analyze the higher order terms in the future.

\subsection{1 $\quad T^{3}$ and $\mathbf{R}^{3}$}

The simplest example of infinite dimensional 3-algebra is given by $T^{3}$ with radius $R$. The basis of functions are parametrized by $\vec{n} \in \mathbf{Z}^{3}$ as (if we take $\mu=(2 \pi R)^{-3}$ )

$$
\chi^{\vec{n}}(\vec{y})=\exp (2 \pi i \vec{n} \cdot \vec{y} / R), \quad \chi_{\vec{n}}(\vec{y})=\exp (-2 \pi i \vec{n} \cdot \vec{y} / R) .
$$

The metric and the structure constants are given by

$$
\begin{aligned}
& h^{\vec{n}_{1} \vec{n}_{2}}=\delta\left(\vec{n}_{1}+\vec{n}_{2}\right), \\
& f^{\vec{n}_{1} \vec{n}_{2} \vec{n}_{3}} \vec{n}_{4}=(2 \pi i / R)^{3} \vec{n}_{1} \cdot\left(\vec{n}_{2} \times \vec{n}_{3}\right) \delta\left(\vec{n}_{1}+\vec{n}_{2}+\vec{n}_{3}-\vec{n}_{4}\right), \\
& f^{\vec{n}_{1} \vec{n}_{2} \vec{n}_{3}, \vec{n}_{4}}=(2 \pi i / R)^{3} \vec{n}_{1} \cdot\left(\vec{n}_{2} \times \vec{n}_{3}\right) \delta\left(\vec{n}_{1}+\vec{n}_{2}+\vec{n}_{3}+\vec{n}_{4}\right) .
\end{aligned}
$$

If we take $R \rightarrow \infty$, we obtain the Lie 3 -algebra associated with $\mathbf{R}^{3}$. The label for the basis becomes continuous and the metric becomes the delta function.

\subsection{2 $S^{3}$}

We introduce four variables $y_{1}, \cdots, y_{4}$ and the Nambu-Poisson bracket defined by

$$
P=-y_{1} \partial_{2} \wedge \partial_{3} \wedge \partial_{4}+y_{2} \partial_{1} \wedge \partial_{3} \wedge \partial_{4}-y_{3} \partial_{1} \wedge \partial_{2} \wedge \partial_{4}+y_{4} \partial_{1} \wedge \partial_{2} \wedge \partial_{3}
$$

If we restrict $C(\mathcal{N})$ to the linear functions of $y_{i}$, it agrees with $\mathcal{A}_{4}$. We impose a constraint $\phi(y):=$ $y_{1}^{2}+y_{2}^{2}+y_{3}^{2}+y_{4}^{2}-1=0$ in $\mathbf{R}^{4}$ which defines $S^{3}$. This restriction is compatible with the Nambu-Poisson bracket in a sense $\left.\left\{\phi(y) f_{1}(y), f_{2}(y), f_{3}(y)\right\}\right|_{\phi(y)=0}=0$ for any $f_{i}(y)$.

Square integrable functions on $S^{3}$ are given by combinations of $y_{1}^{n_{1}} y_{3}^{n_{2}} y_{3}^{n_{3}} y_{4}^{n_{4}}$. By the constraint, whenever powers of $y_{4}$ higher than 2 appears, we can reduce it to zero and one. Therefore the basis of functions are given as

$$
T_{\vec{n}}=y_{1}^{n_{1}} y_{2}^{n_{2}} y_{3}^{n_{3}}, \quad S_{\vec{n}}=y_{1}^{n_{1}} y_{2}^{n_{2}} y_{3}^{n_{3}} y_{4}, \quad\left(n_{i} \geq 0\right) .
$$


The 3-algebra becomes

$$
\begin{aligned}
\left\{T_{\vec{n}}, T_{\vec{m}}, T_{\vec{\ell}}\right\}= & \vec{n} \cdot(\vec{m} \times \vec{\ell}) S_{\vec{n}+\vec{m}+\vec{\ell}-\vec{\rho}}, \\
\left\{T_{\vec{n}}, T_{\vec{m}}, S_{\vec{\ell}}\right\}= & \vec{n} \cdot(\vec{m} \times \vec{\ell})\left(T_{\vec{n}+\vec{m}+\vec{\ell}-\vec{\rho}}-\sum_{i=1}^{3} T_{\vec{n}+\vec{m}+\vec{\ell}-\vec{\rho}+2 \vec{e}_{i}}\right)-\sum_{i=1}^{3} \vec{e}_{i}(\vec{n} \times \vec{m}) T_{\vec{n}+\vec{m}+\vec{\ell}-\vec{\rho}+2 \vec{e}_{i}}, \\
\left\{T_{\vec{n}}, S_{\vec{m}}, S_{\vec{\ell}}\right\}= & \vec{n} \cdot(\vec{m} \times \vec{\ell})\left(S_{\vec{n}+\vec{m}+\vec{\ell}-\vec{\rho}}-\sum_{i=1}^{3} S_{\vec{n}+\vec{m}+\vec{\ell}-\vec{\rho}+2 \vec{e}_{i}}\right)-\sum_{i=1}^{3} \vec{e}_{i}(\vec{n} \times(\vec{m}-\vec{\ell})) S_{\vec{n}+\vec{m}+\vec{\ell}-\vec{\rho}+2 \vec{e}_{i}}, \\
\left\{S_{\vec{n}}, S_{\vec{m}}, S_{\vec{\ell}}\right\}= & \vec{n} \cdot(\vec{m} \times \vec{\ell})\left(T_{\vec{n}+\vec{m}+\vec{\ell}-\vec{\rho}}-2 \sum_{i=1}^{3} T_{\vec{n}+\vec{m}+\vec{\ell}-\vec{\rho}+2 \vec{e}_{i}}+\sum_{i, j} T_{\vec{n}+\vec{m}+\vec{\ell}-\vec{\rho}+2 \vec{e}_{i}+2 \vec{e}_{j}}\right) \\
& -\sum_{i} \vec{e}_{i}(\vec{n} \times \vec{m}+\vec{m} \times \vec{\ell}+\vec{\ell} \times \vec{n})\left(T_{\vec{n}+\vec{m}+\vec{\ell}-\vec{\rho}+2 \vec{e}_{i}}-T_{\vec{n}+\vec{m}+\vec{\ell}-\vec{\rho}+4 \vec{e}_{i}}\right) \\
& +\sum_{i<j}\left(\vec{e}_{i}+\vec{e}_{j}\right)(\vec{n} \times \vec{m}+\vec{m} \times \vec{\ell}+\vec{\ell} \times \vec{n}) T_{\vec{n}+\vec{m}+\vec{\ell}-\vec{\rho}+2 \vec{e}_{i}+2 \vec{e}_{j},}
\end{aligned}
$$

where $\vec{\rho}=\vec{e}_{1}+\vec{e}_{2}+\vec{e}_{3}$.

\section{$3 \quad$ M2 to M5}

In this section we will show that the BLG model with a Nambu-Poisson structure on a 3 dimensional manifold contains the low energy degrees of freedom on an M5-brane. Before going on, let us count the number of degrees of freedom in the bosonic and fermionic sectors in our model. The fermion $\Psi$ is a Majorana spinor in $10+1$ dimensions with a chirality condition, and thus it has 16 real fermionic components, equivalent to 8 bosonic degrees of freedom. For a 5 -brane there are 5 transverse directions corresponding to 5 scalars $X^{i}$. For an ordinary 2 -form gauge field in $6 \mathrm{D}$, there are 6 propagating modes. But since we do not have the usual kinetic term for $A$, but rather a Chern-Simons term, there are only 3 propagating modes. The low energy effective theory of an M5-brane contains the same number of bosonic and fermionic degrees of freedom. But a salient feature of the M5-brane is that the 2-form gauge field is self-dual. Hence our major challenge is to show that the gauge field of the BLG model is equivalent to a self-dual 2-form gauge field.

A comment on the notation: we will use $I . J, K$ to label the transverse directions to the membrane worldvolume $\mathcal{M}$. We decompose this eight dimensional space as a direct product of $\mathcal{N}$ and remaining

5 dimensional space. We use $\dot{\mu}, \dot{\nu}, \dot{\lambda}$ to label $\mathcal{N}$ and $i, j, k$ to label the transverse directions of the M5-brane.

\subsection{Rewriting fields and covariant derivative}

We expand the fields in BL action in terms of a basis $\left\{\chi^{a}(y)\right\}$ of $C(\mathcal{N})$ as

$$
\begin{aligned}
& X^{I}(x, y)=\sum_{a} X_{a}^{I}(x) \chi^{a}(y), \\
& \Psi(x, y)=\sum_{a} \Psi_{a}(x) \chi^{a}(y), \\
& A_{\mu b}(x, y)=\sum_{a} A_{\mu a b}(x) \chi^{a}(y),
\end{aligned}
$$


which show that the original 3 dimensional fields on M2-branes are promoted to 6 dimensional ones. We note that the gauge potential $A_{\mu b}$ still contains the index $b$ which labels the basis of $C(\mathcal{N})$.

The covariant derivative is

$$
D_{\mu} X^{I}(x, y)=\partial_{\mu} X^{I}(x, y)-\left\{\chi^{c}, \chi^{d}, \chi^{b}\right\} A_{\mu c d}(x) X_{b}^{I}=\partial_{\mu} X^{I}(x, y)-\left\{A_{\mu d}(x, y), \chi^{d}(y), X^{I}(x, y)\right\} .
$$

\subsection{Action}

In the following, we will rewrite the M2 action in terms of the six dimensional fields defined in (23$25)$. For this purpose, we decompose the summation in $I, J, K$ to the summation in $\dot{\mu}, \dot{\nu}, \dot{\lambda}$ and $i, j, k$. Without loss of generality, we will take ${ }^{1}$

$$
P^{\dot{\mu} \dot{\nu} \dot{\lambda}}=\epsilon^{\dot{\mu} \dot{\nu} \dot{\lambda}}
$$

and

$$
\langle f, g\rangle=\int d^{3} y f g
$$

\subsubsection{Potential term}

$$
\begin{aligned}
L_{V} & =-\frac{1}{12}\left\langle\left[X^{I}, X^{J}, X^{K}\right],\left[X^{I}, X^{J}, X^{K}\right]\right\rangle \\
& =-\frac{1}{12}\left\langle\left(\left[X^{\dot{\mu}}, X^{\dot{\nu}}, X^{\dot{\lambda}}\right]^{2}+3\left[X^{\dot{\mu}}, X^{\dot{\nu}}, X^{i}\right]^{2}+3\left[X^{\dot{\mu}}, X^{i}, X^{j}\right]^{2}+\left[X^{i}, X^{j}, X^{k}\right]^{2}\right)\right\rangle .
\end{aligned}
$$

As in matrix models [23-25], the first trick to reorganize the 6 dimensional fields is to rewrite

$$
X^{\dot{\mu}}(x, y)=y^{\dot{\mu}}+A^{\dot{\mu}}(x, y) .
$$

Thus

$$
\begin{aligned}
\left\langle\left[X^{\dot{\mu}}, X^{\dot{\nu}}, X^{\dot{\lambda}}\right]^{2}\right\rangle= & \left(\epsilon^{\dot{\mu} \dot{\nu} \dot{\lambda}}\right)^{2}+12\left(\partial^{\dot{\mu}} A^{\dot{\mu}}\right)+12\left(\partial^{\dot{\mu}} A^{\dot{\mu}}\right)^{2}-6\left(\partial^{\dot{\mu}} A^{\dot{\nu}} \partial^{\dot{\nu}} A^{\dot{\mu}}\right)+\mathcal{O}\left(A^{3}\right) \\
= & 6+6 \epsilon^{\dot{\mu} \dot{\nu} \dot{\lambda}} \partial_{\dot{\mu}} A_{\dot{\nu} \dot{\lambda}}+3\left(\epsilon^{\dot{\mu} \dot{\nu} \dot{\lambda}} \partial_{\dot{\mu}} A_{\dot{\nu} \dot{\lambda}}\right)^{2}-\frac{3}{2} \epsilon^{\dot{\nu} \dot{\lambda} \dot{\omega}} \epsilon^{\dot{\mu} \dot{\pi} \dot{\epsilon}} \partial_{\dot{\mu}} A_{\dot{\lambda} \dot{\omega}} \partial_{\dot{\nu}} A_{\dot{\pi} \dot{\epsilon}} \\
& +\mathcal{O}\left(A^{3}\right),
\end{aligned}
$$

where

$$
A_{\dot{\mu} \dot{\nu}} \equiv \epsilon_{\dot{\mu} \dot{\mu} \dot{\lambda}} A^{\dot{\lambda}} .
$$

The gauge transformation of the gauge field $A^{\dot{\mu}}$ is given by

$$
\delta A^{\dot{\mu}}(x, y)=\sum_{\alpha}\left[f_{1 \alpha}(x, y), f_{2 \alpha}(x, y), y^{\dot{\mu}}+A^{\dot{\mu}}\right]=\epsilon^{\dot{\mu} \dot{\nu} \dot{\lambda}} \sum_{\alpha} \partial_{\dot{\nu}} f_{1 \alpha} \partial_{\dot{\lambda}} f_{2 \alpha}+\mathcal{O}(A) .
$$

The linear part of the transformation of $A_{\dot{\mu} \dot{\nu}}$ becomes

$$
\delta A=d \Lambda+\mathcal{O}(A), \quad A \equiv \frac{1}{2} A_{\dot{\mu} \dot{\nu}} d y^{\dot{\mu}} d y^{\dot{\nu}}, \quad \Lambda=\sum_{\alpha} f_{1 \alpha} d f_{2 \alpha},
$$

\footnotetext{
${ }^{1}$ Of course, this is possible only locally on each coordinate patch for generic $\mathcal{N}$.
} 
as that of a standard 2-form gauge field. Thus at the quadratic level of the action, we define the field strength of $A$ as

$$
F_{\dot{\mu} \dot{\nu} \dot{\lambda}}=\partial_{\dot{\mu}} A_{\dot{\nu} \dot{\lambda}}+\partial_{\dot{\nu}} A_{\dot{\lambda} \dot{\nu}}+\partial_{\dot{\lambda}} A_{\dot{\mu} \dot{\nu}}
$$

Since $\dot{\mu}, \dot{\nu}, \dot{\lambda}=1,2,3$, we have

$$
F_{\dot{\mu} \dot{\nu} \dot{\lambda}}=\frac{1}{2} \epsilon_{\dot{\mu} \dot{\nu} \dot{\lambda}} \epsilon^{\dot{\pi} \dot{\omega} \dot{\epsilon}} \partial_{\dot{\pi}} A_{\dot{\omega} \dot{\epsilon}} .
$$

Now we try to rewrite the quadratic part of the potential in terms of the field strength $F$. The first term in (31) is a constant. The 2nd term is a total derivative, unless $F$ is a constant. The 3rd term is

$$
3\left(\epsilon^{\dot{\mu} \dot{\mu} \dot{\lambda}} \partial_{\dot{\mu}} A_{\dot{\nu} \dot{\lambda}}\right)^{2}=2 F_{\dot{\mu} \dot{\nu} \dot{\lambda}}{ }^{2} .
$$

After integration by parts, the 4 th term becomes

$$
-\frac{3}{2}\left(\epsilon^{\dot{\mu} \dot{\mu} \dot{\lambda}} \partial_{\dot{\mu}} A_{\dot{\nu} \dot{\lambda}}\right)^{2}=-F_{\dot{\mu} \dot{\nu} \dot{\lambda}}{ }^{2} .
$$

Thus, altogether, we find up to total derivatives ${ }^{2}$

$$
\left\langle\left[X^{\dot{\mu}}, X^{\dot{\nu}}, X^{\dot{\lambda}}\right]^{2}\right\rangle=\int d^{3} y\left(F_{\dot{\mu} \dot{\lambda} \dot{\nu}}^{2}+\text { constant }+\mathcal{O}\left(A^{3}\right)\right) .
$$

We also have the 2 nd term in (29) given by

$$
-\frac{1}{4}\left[X^{\dot{\mu}}, X^{\dot{\nu}}, X^{i}\right]^{2}=-\frac{1}{2}\left(\partial_{\dot{\mu}} X^{i}\right)^{2}+\mathcal{O}\left(A X^{2}\right) .
$$

The 3rd and 4th terms in (29) have no quadratic terms.

\subsubsection{Fermion kinetic term}

The kinetic term for the fermion is easy to compute:

$$
\frac{i}{4}\left\langle\bar{\Psi}, \Gamma_{I J}\left[X^{I}, X^{J}, \Psi\right]\right\rangle \sim \frac{i}{4}\left\langle\bar{\Psi}, \Gamma_{\dot{\mu} \dot{\nu}}\left[y^{\dot{\mu}}, y^{\dot{\nu}}, \Psi\right]\right\rangle=\frac{i}{4} \epsilon^{\dot{\mu} \dot{\nu}} \dot{\lambda}\left\langle\bar{\Psi}, \Gamma_{\dot{\mu} \dot{\nu}} \partial_{\dot{\lambda}} \Psi\right\rangle=\frac{i}{2}\left\langle\bar{\Psi}, \Gamma^{\dot{\lambda}} \partial_{\dot{\lambda}} \Psi\right\rangle .
$$

\subsubsection{Chern-Simons term}

The Chern-Simons term in the BL action is

$$
L_{C S}=\frac{1}{2} \epsilon^{\mu \nu \lambda}\left(f^{a b c d} A_{\mu a b} \partial_{\nu} A_{\lambda c d}+\frac{2}{3} f_{g}^{c d a} f^{e f g b} A_{\mu a b} A_{\nu c d} A_{\lambda e f}\right) .
$$

It can be rewritten as

$$
\begin{aligned}
L_{C S}= & \frac{1}{2} \epsilon^{\mu \nu \lambda}\left(\left\langle\left\{\chi^{a}, \chi^{b}, \chi^{c}\right\}, \chi^{d}\right\rangle A_{\mu a b} \partial_{\nu} A_{\lambda c d}\right. \\
& \left.\quad+\frac{2}{3}\left\langle\left\{\chi^{c}, \chi^{d}, \chi^{a}\right\}, \chi_{g}\right\rangle\left\langle\left\{\chi^{e}, \chi^{f}, \chi^{g}\right\}, \chi^{b}\right\rangle A_{\mu a b} A_{\nu c d} A_{\lambda e f}\right) \\
= & \frac{1}{2} \epsilon^{\mu \nu \lambda}\left(\left\langle\left\{A_{\mu b}, \chi^{b}, \partial_{\nu} A_{\lambda d}\right\}, \chi^{d}\right\rangle+\frac{2}{3}\left\langle\left\{A_{\nu d}, \chi^{d}, A_{\mu b}\right\}, \chi_{g}\right\rangle\left\langle\left\{A_{\lambda f}, \chi^{f}, \chi^{g}\right\}, \chi^{b}\right\rangle\right) .
\end{aligned}
$$

\footnotetext{
${ }^{2}$ Keeping all the total derivatives, it is

$$
\left\langle\left[X^{\dot{\mu}}, X^{\dot{\nu}}, X^{\dot{\lambda}}\right]^{2}\right\rangle=\int d^{3} y\left[\left(1+\frac{1}{6} \epsilon^{\dot{\mu} \dot{\nu}} F_{\dot{\mu} \dot{\nu} \dot{\lambda}}\right)^{2}+\partial_{\dot{\mu}}\left(A^{\dot{\mu}} \partial_{\dot{\nu}} A^{\dot{\nu}}-A^{\dot{\nu}} \partial_{\dot{\nu}} A^{\dot{\mu}}\right)+\mathcal{O}\left(A^{3}\right)\right] .
$$
}


Let us now focus on the quadratic term. Here we need to introduce the second trick. In the choice of the basis $\chi^{a}$, we pick the first three as

$$
\chi^{\dot{\mu}}(y)=y^{\dot{\mu}} .
$$

The rest of the basis $\chi^{a}$ correspond to higher oscillations modes. ${ }^{3}$ They are ignored for the purpose of this paper, which is to identify the physical degrees of freedom of the low energy effective M5-brane theory.

For these modes, we change the label $a$ to the label $\dot{\mu}$ of the coordinates of $\mathcal{N}$. We pick up the corresponding mode in $A_{\mu a}$ (rewritten as $A_{\mu \dot{\mu}}$ ) in the quadratic terms in $L_{C S}$ and find

$$
L_{C S}^{q u a d}=-\frac{1}{2} \int d^{3} y \epsilon^{\mu \nu \lambda} \epsilon^{\dot{\mu} \dot{\nu} \dot{\lambda}} \partial_{\mu} A_{\nu \dot{\mu}}(x, y) \partial_{\dot{\nu}} A_{\lambda \dot{\lambda}}(x, y)+\cdots .
$$

The dots $\cdots$ represent the terms which involve $A_{\mu a}$ 's for higher oscillation modes. In the following we will ignore them for simplicity.

\subsubsection{Kinetic term for $X$}

The kinetic terms for $X^{I}$ 's are

$$
\begin{aligned}
\left(D_{\mu} X^{I}\right)^{2} & =\left(D_{\mu} X^{\dot{\nu}}\right)^{2}+\left(D_{\mu} X^{i}\right)^{2} \\
& =\left(\partial_{\mu} A^{\dot{\nu}}-\left\{A_{\mu \dot{\lambda}}, y^{\dot{\lambda}}, y^{\dot{\nu}}\right\}\right)^{2}+\left(\partial_{\mu} X^{i}\right)^{2}+\cdots \\
& =\left(\frac{1}{2} \epsilon^{\dot{\nu} \dot{\mu} \dot{\lambda}} \partial_{\mu} A_{\dot{\mu} \dot{\lambda}}-\epsilon^{\dot{\lambda} \dot{\mu} \dot{\mu}} \partial_{\dot{\mu}} A_{\mu \dot{\lambda}}\right)^{2}+\left(\partial_{\mu} X^{i}\right)^{2}+\cdots \\
& =\frac{1}{2}\left(F_{\mu \dot{\nu} \dot{\lambda}}\right)^{2}+\left(\partial_{\mu} X^{i}\right)^{2}+\cdots,
\end{aligned}
$$

where we defined the field strength of $A$

$$
F_{\mu \dot{\nu} \dot{\lambda}} \equiv \partial_{\mu} A_{\dot{\nu} \dot{\lambda}}-\partial_{\dot{\nu}} A_{\mu \dot{\lambda}}+\partial_{\dot{\lambda}} A_{\mu \dot{\nu}}
$$

Here again, $\cdots$ represents those terms involving $A_{\mu a}(a \neq 1,2,3)$ and higher order terms which we ignore in the analysis in the following.

\subsection{Equivalence to M5-brane low energy theory}

Collecting all relevant terms, the quadratic part of the BL Lagrangian is

$$
\begin{aligned}
L^{\text {quad }}=-\frac{1}{2}[ & \left.\left(\partial_{\mu} X^{i}\right)^{2}+\left(\partial_{\dot{\mu}} X^{i}\right)^{2}\right]+\frac{i}{2}\left\langle\bar{\Psi},\left(\Gamma^{\mu} \partial_{\mu}+\Gamma^{\dot{\mu}} \partial_{\dot{\mu}}\right) \Psi\right\rangle \\
& -\frac{1}{4} F_{\mu \dot{\nu} \dot{\lambda}}{ }^{2}-\frac{1}{12} F_{\dot{\mu} \dot{\nu} \dot{\lambda}}{ }^{2}-\frac{1}{2} \epsilon^{\mu \nu \lambda} \epsilon^{\dot{\mu} \dot{\nu} \dot{\lambda}} \partial_{\mu} A_{\nu \dot{\mu}} \partial_{\dot{\nu}} A_{\lambda \dot{\lambda}} .
\end{aligned}
$$

The first two terms on the right hand side are the standard kinetic terms for free fields living on a 6 dimensional space. They agree with what we expect for an M5-brane. We will now focus our attention on the gauge fields.

\footnotetext{
${ }^{3}$ The zero mode $\chi^{0}=1$ has no contribution to the CS action since the Nambu-Poisson bracket vanishes whenever it is present.
} 
The last term in the Lagrangian can be rewritten as

$$
-\frac{1}{2} \epsilon^{\mu \nu \lambda} \epsilon^{\dot{\mu} \dot{\nu} \dot{\lambda}} \partial_{\mu} A_{\nu \dot{\mu}} \partial_{\dot{\nu}} A_{\lambda \dot{\lambda}}=-\frac{1}{8} \epsilon^{\mu \nu \lambda} \epsilon^{\dot{\mu} \dot{\nu} \dot{\lambda}} F_{\mu \dot{\mu} \dot{\nu}} F_{\nu \lambda \dot{\lambda}}-\frac{1}{4} \epsilon^{\mu \nu \lambda} \epsilon^{\dot{\mu} \dot{\nu} \dot{\lambda}} \partial_{\mu}\left(A_{\dot{\mu} \dot{\nu}} \partial_{\nu} A_{\lambda \dot{\lambda}}\right) .
$$

where

$$
F_{\nu \lambda \dot{\lambda}} \equiv \partial_{\nu} A_{\lambda \dot{\lambda}}-\partial_{\lambda} A_{\nu \dot{\lambda}}
$$

This is different from the expression of a field strength for an ordinary 2-form gauge field because it misses the term $\partial_{\dot{\lambda}} A_{\nu \lambda}$. Hence $A_{\dot{\mu} \dot{\nu}}$ is a 2-form gauge field, and $A_{\mu \dot{\mu}}$ is a 1 -form gauge field with an addition index $\dot{\mu}$. The gauge transformations are

$$
\begin{aligned}
\delta A_{\dot{\mu} \dot{\nu}}(x, y) & =\partial_{\dot{\mu}} \Lambda_{\dot{\nu}}(x, y)-\partial_{\dot{\nu}} \Lambda_{\dot{\mu}}(x, y) \\
\delta A_{\mu \dot{\mu}}(x, y) & =\partial_{\mu} \Lambda_{\dot{\mu}}(x, y) .
\end{aligned}
$$

Compared with a 2 -form gauge field on a 6 -dimensional spacetime, we are missing the components $A_{\mu \nu}$ and the gauge transformation corresponding to the other 3 components of the gauge parameter $\Lambda_{\mu}$. We will see below how they automatically appear when we analyze the equations of motion in more detail.

One can rewrite the gauge field relevant terms in (49) as

$$
L_{A}^{q u a d}=-\frac{1}{4} F_{\mu \dot{\mu} \dot{\nu}}\left(F_{\mu \dot{\mu} \dot{\nu}}+\tilde{F}_{\mu \dot{\mu} \dot{\nu}}\right)-\frac{1}{12} F_{\dot{\mu} \dot{\nu} \dot{\lambda}}^{2},
$$

where $\tilde{F}$ is the Hodge dual of the field strength defined by

$$
\tilde{F}_{\underline{\mu} \underline{\nu \lambda}}=\frac{1}{6} \epsilon_{\underline{\mu} \underline{\nu \Lambda \kappa \sigma \underline{\rho}}} F_{\underline{\kappa \sigma} \underline{\rho}} .
$$

Here $\underline{\mu}, \underline{\nu}, \underline{\lambda}=0,1, \cdots, 5$ are the collective indices for both $\mu$ and $\dot{\mu}$. In particular,

$$
\tilde{F}_{\mu \nu \dot{\mu}}=-\frac{1}{2} \epsilon_{\mu \nu \lambda} \epsilon^{\dot{\mu} \dot{\nu}} F_{\lambda \dot{\nu} \dot{\lambda}} .
$$

The minus sign on the right hand side of (56) comes from $\epsilon_{\mu \nu \dot{\mu} \lambda \dot{\nu} \dot{\lambda}}=-\epsilon_{\mu \nu \lambda \dot{\mu} \dot{\nu} \dot{\lambda}}=-\epsilon_{\mu \nu \lambda} \epsilon_{\dot{\mu} \dot{\nu} \dot{\lambda}}$.

The equations of motion derived from (54) are

$$
\begin{gathered}
\partial_{\underline{\mu}} F_{\mu \dot{\mu} \dot{\nu}}=0, \\
\partial_{\dot{\nu}} F_{\dot{\nu} \mu \dot{\mu}}+\partial_{\nu} \tilde{F}_{\nu \mu \dot{\mu}}=0 .
\end{gathered}
$$

Let us now show that this set of equations of motion (57), (58) is equivalent to the free field theory of a self-dual 2-form gauge field in 6 dimensions. First we focus on (58). Combined with the Bianchi identity (recall that Hodge dual exchanges equation of motion with Bianchi identity)

$$
\partial_{\nu} \tilde{F}_{\nu \mu \dot{\mu}}+\partial_{\dot{\nu}} \tilde{F}_{\dot{\nu} \mu \dot{\mu}}=0
$$

eq. (58) gives

$$
\partial_{\dot{\nu}}\left(F_{\mu \dot{\mu} \dot{\nu}}-\tilde{F}_{\mu \dot{\mu} \dot{\nu}}\right)=0 .
$$

Hence there exists a 1-form field $B$ such that

$$
F_{\mu \dot{\mu} \dot{\nu}}-\tilde{F}_{\mu \dot{\mu} \dot{\nu}}=\epsilon_{\dot{\mu} \dot{\nu} \dot{\lambda}} \partial_{\dot{\lambda}} B_{\mu} .
$$


Next we consider the equation of motion (57). Using (61), we find that (57) implies

$$
\partial_{\dot{\lambda}} F_{\dot{\lambda} \dot{\mu} \dot{\nu}}=-\partial_{\lambda} F_{\lambda \dot{\mu} \dot{\nu}}=\frac{1}{2} \epsilon_{\mu \nu \lambda} \epsilon_{\dot{\mu} \dot{\nu} \dot{\lambda}} \partial_{\lambda} F_{\mu \nu \dot{\lambda}}-\epsilon_{\dot{\mu} \dot{\nu} \dot{\lambda}} \partial_{\lambda} \partial_{\dot{\lambda}} B_{\lambda}=-\epsilon_{\dot{\mu} \dot{\nu} \dot{\lambda}} \partial_{\dot{\lambda}} \partial_{\lambda} B_{\lambda} .
$$

Since $\dot{\mu}, \dot{\nu}, \dot{\lambda}$ can only take 3 different values, we have

$$
F_{\dot{\mu} \dot{\nu} \dot{\lambda}}=\frac{1}{6} \epsilon_{\dot{\mu} \dot{\nu} \dot{\lambda}} \epsilon_{\dot{\kappa} \dot{\sigma} \dot{\rho}} F_{\dot{\kappa} \dot{\sigma} \dot{\rho}}
$$

and hence it follows from (62) that

$$
\partial_{\dot{\lambda}}\left(F_{\dot{\kappa} \dot{\sigma} \dot{\rho}}+\epsilon_{\dot{\kappa} \dot{\sigma} \dot{\rho}} \partial_{\lambda} B_{\lambda}\right)=0 .
$$

This is solved by

$$
F_{\dot{\kappa} \dot{\sigma} \dot{\rho}}+\epsilon_{\dot{\kappa} \dot{\sigma} \dot{\rho}} \partial_{\lambda} B_{\lambda}=f(x) \epsilon_{\dot{\kappa} \tilde{\sigma} \dot{\rho}},
$$

where $f(x)$ is independent of $y$. We can set $f(x)=0$ by absorbing it in $B_{\mu}$. Then the $B_{\mu}$ 's are shifted by functions of $x$, but this will not change the defining equation (61) of $B_{\mu}$. Thus, by suitably choosing $B_{\mu}$, we have

$$
F_{\dot{\kappa} \dot{\sigma} \dot{\rho}}+\epsilon_{\dot{\kappa} \dot{\sigma} \dot{\rho}} \partial_{\lambda} B_{\lambda}=0 .
$$

Eqs. (61) and (66) allow us to define a self-dual 2-form gauge field $B_{\mu \nu}$ as

$$
B_{\mu \nu}=-\epsilon_{\mu \nu \lambda} B_{\lambda}, \quad B_{\mu \dot{\mu}}=A_{\mu \dot{\mu}}, \quad B_{\dot{\mu} \dot{\nu}}=A_{\dot{\mu} \dot{\nu}}
$$

The field strength $d B$ is denoted by $H$ and its components are

$$
\begin{aligned}
H_{\mu \nu \lambda} & =\partial_{\mu} B_{\nu \lambda}+\partial_{\nu} B_{\lambda \mu}+\partial_{\lambda} B_{\mu \nu}=-\epsilon_{\mu \nu \lambda} \partial_{\rho} B_{\rho}, \\
H_{\mu \nu \dot{\mu}} & =\partial_{\mu} B_{\nu \dot{\mu}}-\partial_{\nu} B_{\mu \dot{\mu}}-\epsilon_{\mu \nu \lambda} \partial_{\dot{\mu}} B_{\lambda}=F_{\mu \nu \dot{\mu}}-\epsilon_{\mu \nu \lambda} \partial_{\dot{\mu}} B_{\lambda}, \\
H_{\mu \dot{\mu} \dot{\nu}} & =\partial_{\mu} B_{\dot{\mu} \dot{\nu}}-\partial_{\dot{\mu}} B_{\mu \dot{\nu}}+\partial_{\dot{\nu}} B_{\mu \dot{\mu}}=F_{\mu \dot{\mu} \dot{\nu}}, \\
H_{\dot{\mu} \dot{\nu} \dot{\lambda}} & =\partial_{\dot{\mu}} B_{\dot{\nu} \dot{\lambda}}+\partial_{\dot{\nu}} B_{\dot{\lambda} \dot{\mu}}+\partial_{\dot{\lambda}} B_{\dot{\mu} \dot{\nu}}=F_{\dot{\mu} \dot{\nu} \dot{\lambda}} .
\end{aligned}
$$

For a self-dual field theory, the 3 -form field strength satisfies

$$
\begin{array}{rlrl}
H_{\mu \nu \lambda} & =\frac{1}{6} \epsilon_{\mu \nu \lambda} \epsilon^{\dot{\mu} \dot{\nu} \dot{\lambda}} H_{\dot{\mu} \dot{\nu} \dot{\lambda}}, & H_{\dot{\mu} \dot{\nu} \dot{\lambda}} & =\frac{1}{6} \epsilon_{\mu \nu \lambda} \epsilon^{\dot{\mu} \dot{\nu} \dot{\lambda}} H_{\mu \nu \lambda}, \\
H_{\mu \nu \dot{\mu}}=-\frac{1}{2} \epsilon_{\mu \nu \lambda} \epsilon^{\dot{\mu} \dot{\nu} \dot{\lambda}} H_{\lambda \dot{\nu} \dot{\lambda}}, & H_{\mu \dot{\mu} \dot{\nu}}=\frac{1}{2} \epsilon_{\mu \nu \lambda} \epsilon^{\dot{\mu} \dot{\lambda} \dot{\lambda}} H_{\nu \lambda \dot{\lambda}} .
\end{array}
$$

(The two equations on the same line are equivalent.) It is straightforward to check that the self-duality conditions are guaranteed by (61) and (66). Thus we have proven that (61) and (66) are equivalent to the free field theory of a self-dual 2-form gauge field in 6 dimensions. The Lagrangian (54) can thus be understood as a Lagrangian for a self-dual 2-form gauge field in $6 \mathrm{D}$, and it is different from such Lagrangians in the literature [20,21].

\section{Remarks}

One may wonder the possibility of constructing other $\mathrm{M} p$-branes (which should not exist) in $\mathrm{M}$ theory from multiple M2-branes. However, even if we had considered a higher dimensional manifold $\mathcal{N}$ with 
Nambu-Poisson structure, due to the decomposability [17] of the Nambu-Poisson bracket, locally one can always choose 3 coordinates $\left\{y^{1}, y^{2}, y^{3}\right\}$ in terms of which the bracket is simply

$$
\{f, g, h\}=\epsilon^{\dot{\mu} \dot{\nu} \dot{\lambda}} \partial_{\dot{\mu}} f \partial_{\dot{\nu}} g \partial_{\dot{\lambda}} h .
$$

Hence the rest of the coordinates $\left(y^{a}\right.$ for $a>3$ ) of $\mathcal{N}$ will not induce derivatives or gauge field components. There can never be more than 3 of the $X^{I}$ 's turning into covariant derivatives. The decomposability of the Nambu-Poisson bracket is thus the mathematical basis of why there are no other $\mathrm{M} p$-branes with $p \neq 5$.

In order to understand this statement, it may be instructive to consider a straightforward extension,

$$
P=\partial_{1} \wedge \partial_{2} \wedge \partial_{3}+\partial_{4} \wedge \partial_{5} \wedge \partial_{6}
$$

which would give us a theory on M8-brane. This does not work however since this bracket does NOT satisfy the fundamental identity! One may easily confirm this by examining

$$
\begin{aligned}
& \left\{y_{1} y_{4}, y_{2},\left\{y_{3}, y_{5}, y_{6}\right\}\right\}=0, \quad \text { but } \\
& \left\{\left\{y_{1} y_{4}, y_{2}, y_{3}\right\}, y_{5}, y_{6}\right\}+\left\{y_{3},\left\{y_{1} y_{4}, y_{2}, y_{5}\right\}, y_{6}\right\}+\left\{y_{3}, y_{5},\left\{y_{1} y_{4}, y_{2}, y_{6}\right\}\right\}=1 .
\end{aligned}
$$

The fact that the fundamental identity is so restrictive is helpful here to restrict the branes of M-theory to M2 and M5.

The Nambu-Poisson tensor $P_{\mu \dot{\mu} \dot{\lambda}}$ is reminiscent of the Poisson tensor $\theta_{\dot{\mu} \dot{\nu}}$ which appears on a $\mathrm{D} p$ brane world volume when there is a constant $B$-field background [26]. In both the weak and strong $B$-field limit, the noncommutative structure on the D-brane world volume can be approximated by the Poisson structure. By analogy, we suspect that the M5-brane action presented above corresponds to a weak or strong $C$-field limit with $C_{\dot{\mu} \dot{\nu} \dot{\lambda}}$ turned on. For a finite value of $C_{\dot{\mu} \dot{\nu} \dot{\lambda}}$, we expect that the Nambu-Poisson bracket to be replaced by a quantum version. In [27] we proposed a quantum Nambu bracket by examining open membrane scattering amplitudes in the large $C$-field background. However, the fundamental identity is not preserved by the quantum bracket. If it is the correct formulation, one should interpret it as the Nambu bracket after gauge fixing $A_{\mu \dot{\nu}}=0$.

There are obviously many things to be clarified in the future. In this paper we consider only the quadratic part of the Lagrangian and ignored higher terms and also the components for $A_{\mu a}$ for $a \neq 1,2,3$. To study the precise role played by them would be essential to understand the precise relation between M2 and M5. It will also be possible to study the opposite direction, to understand M2 from M5 brane action [20,21]. Since M5 action is non-polynomial DBI type action, we expect to have a similar non-polynomial action which generalizes (1). Since the higher powers of the generators of Lie 3-algebra will be inevitable, one needs to understand global structure associated with a given Lie 3-algebra. This is related to the problem of the quantum Nambu-bracket, a notoriously difficult problem but many attempts $[13,15,27]$ have been made. We hope that our study here would provide a good hint to this problem.

\section{Acknowledgment}

We appreciate partial financial support from Japan-Taiwan Joint Research Program provided by Interchange Association (Japan) by which this collaboration is made possible. 
The authors thank Kazuyuki Furuuchi, Yosuke Imamura Takeo Inami, Hsien-chung Kao, Xue-Yen Lin, Darren Sheng-Yu Shih, and Wen-Yu Wen for helpful discussions. P.-M. H. is supported in part by the National Science Council, and the National Center for Theoretical Sciences, Taiwan, R.O.C. Y. M. is partially supported by Grant-in-Aid (\#20540253) from the Japan Ministry of Education, Culture, Sports, Science and Technology.

\section{References}

[1] J. Bagger and N. Lambert, "Modeling multiple M2's," Phys. Rev. D 75, 045020 (2007) [arXiv:hepth/0611108].

[2] J. Bagger and N. Lambert, "Gauge Symmetry and Supersymmetry of Multiple M2-Branes," Phys. Rev. D 77, 065008 (2008) [arXiv:0711.0955 [hep-th]].

[3] J. Bagger and N. Lambert, "Comments On Multiple M2-branes," JHEP 0802, 105 (2008) [arXiv:0712.3738 [hep-th]].

[4] A. Gustavsson, "Algebraic structures on parallel M2-branes," arXiv:0709.1260 [hep-th].

[5] A. Gustavsson, "Selfdual strings and loop space Nahm equations," arXiv:0802.3456 [hep-th].

[6] V. T. Filippov, "n-Lie algebras," Sib. Mat. Zh.,26, No. 6, 126140 (1985).

[7] Y. Kawamura, "Cubic matrix, generalized spin algebra and uncertainty relation," Prog. Theor. Phys. 110, 579 (2003) [arXiv:hep-th/0304149].

[8] J. Figueroa-O'Farrill and G. Papadopoulos, "Pluecker-type relations for orthogonal planes," arXiv:math/0211170.

[9] M. A. Bandres, A. E. Lipstein and J. H. Schwarz, "N = 8 Superconformal Chern-Simons Theories," arXiv:0803.3242 [hep-th].

[10] P. M. Ho, R. C. Hou and Y. Matsuo, "Lie 3-Algebra and Multiple M2-branes," arXiv:0804.2110 [hep-th].

[11] G. Papadopoulos, "M2-branes, 3-Lie Algebras and Plucker relations," arXiv:0804.2662 [hep-th].

[12] J. P. Gauntlett and J. B. Gutowski, "Constraining Maximally Supersymmetric Membrane Actions," arXiv:0804.3078 [hep-th]. 
[13] H. Awata, M. Li, D. Minic and T. Yoneya, "On the quantization of Nambu brackets," JHEP 0102, 013 (2001) [arXiv:hep-th/9906248].

[14] Y. Nambu, "Generalized Hamiltonian dynamics," Phys. Rev. D 7, 2405 (1973).

[15] For example, L. Takhtajan, "On Foundation Of The Generalized Nambu Mechanics (Second Version)," Commun. Math. Phys. 160, 295 (1994) [arXiv:hep-th/9301111]; G. Dito, M. Flato, D. Sternheimer and L. Takhtajan, "Deformation quantization and Nambu mechanics," Commun. Math. Phys. 183, 1 (1997) [arXiv:hep-th/9602016]: J. Hoppe, "On M-Algebras, the Quantisation of Nambu-Mechanics, and Volume Preserving Diffeomorphisms," Helv. Phys. Acta 70, 302 (1997) [arXiv:hep-th/9602020]; E. Bergshoeff, D. S. Berman, J. P. van der Schaar and P. Sundell, "A noncommutative M-theory five-brane," Nucl. Phys. B 590, 173 (2000) [arXiv:hep-th/0005026]; S. Kawamoto and N. Sasakura; "Open membranes in a constant C-field background and noncommutative boundary strings," JHEP 0007, 014 (2000) [arXiv:hep-th/0005123]; Y. Matsuo and Y. Shibusa, "Volume preserving diffeomorphism and noncommutative branes," JHEP 0102, 006 (2001) [arXiv:hep-th/0010040]; Y. Kawamura, "Cubic matrix, Nambu mechanics and beyond," Prog. Theor. Phys. 109, 153 (2003) [arXiv:hep-th/0207054]; B. Pioline, "Comments on the topological open membrane," Phys. Rev. D 66, 025010 (2002) [arXiv:hep-th/0201257]; A. De Castro, M. P. Garcia del Moral, I. Martin and A. Restuccia, "M5-brane as a Nambu-Poisson geometry of a multi D1-brane theory," Phys. Lett. B 584, 171 (2004) [arXiv:hep-th/0306094]; T. L. Curtright and C. K. Zachos, "Deformation quantization of superintegrable systems and Nambu mechanics," New J. Phys. 4, 83 (2002) [arXiv:hep-th/0205063]; T. Curtright and C. K. Zachos, "Classical and quantum Nambu mechanics," Phys. Rev. D 68, 085001 (2003) [arXiv:hep-th/0212267];

A review article is, D. S. Berman, "M-theory branes and their interactions," arXiv:0710.1707 [hep-th].

[16] M. Schlesinger, J. D. Stasheff, "The Lie algebra structure of tangent cohomology and deformation theory," J. Pure Appl. Algebra 38 (1985), 313. P. Hanlon, M. L. Wachs, "On Lie $k$-algebras," Adv. in Math. 113 (1995), 206. J. A. Azcárraga, A. M. Perelomov, J. C. Pérez Bueno, "New generalized Poisson structures," J. Phys. A29 (1996), 627. "n-ary Lie and associative algebras," Redn. Sem. Math. Univ. Pol. Torino 53 (1996), 373. J. A. Azcárraga, J. M. Izquierdo, J. C. Pérez 
Bueno, "On the higher order generalizations of Poisson structures", J. Phys. A30 (1997), L607. R. Ibáñez, M. de León, J. C. Marrero, D. Martín de Diego, "Dynamics of generalized Poisson and Nambu-Poisson brackets," J. Math. Phys. 38 (1997), 2332. P. W. Michor, A. M. Vinogradov, "n-ary Lie and associative algebras," Rend. Sem. Mat. Torino, 53 (1996), 373.

[17] R. Weitzenbök, "Invariantentheorie," P. Noordhoff, Gröningen, 1923. Ph. Gautheron, "Some remarks concerning Nambu mechanics," Lett. in Math. Phys. 37 (1996), 103. D. Alekseevsky, P. Guha, "On Decomposability of Nambu-Poisson Tensor," Acta. Math. Univ. Commenianae 65 (1996), 1. R. Ibánez, M. de León, J. C. Marrero, D. M. de Diego, "Dynamics of generalized Poisson and Nambu-Poisson brackets," J. of Math. Physics 38 (1997), 2332. N. Nakanishi, "On Nambu-Poisson Manifolds," Reviews in Mathematical Physics 10 (1998), 499. G. Marmo, G. Vilasi, A. M. Vinogradov, "The local structure of n-Poisson and n-Jacobi manifolds," J. Geom. Physics 25 (1998), 141.

[18] I. Vaisman, "A survey on Nambu-Poisson brackets," Acta. Math. Univ. Comenianae 2 (1999), 213.

[19] In addition to [9-12], see: S. Mukhi and C. Papageorgakis, "M2 to D2," arXiv:0803.3218 [hepth]. D. S. Berman, L. C. Tadrowski and D. C. Thompson, "Aspects of Multiple Membranes," arXiv:0803.3611 [hep-th]. M. Van Raamsdonk, "Comments on the Bagger-Lambert theory and multiple M2-branes," arXiv:0803.3803 [hep-th]. A. Morozov, "On the Problem of Multiple M2 Branes," arXiv:0804.0913 [hep-th]. N. Lambert and D. Tong, "Membranes on an Orbifold," arXiv:0804.1114 [hep-th]. J. Distler, S. Mukhi, C. Papageorgakis and M. Van Raamsdonk, "M2branes on M-folds," arXiv:0804.1256 [hep-th]. U. Gran, B. E. W. Nilsson and C. Petersson, "On relating multiple M2 and D2-branes," arXiv:0804.1784 [hep-th]. J. Gomis, A. J. Salim and F. Passerini, "Matrix Theory of Type IIB Plane Wave from Membranes," arXiv:0804.2186 [hepth]. E. A. Bergshoeff, M. de Roo and O. Hohm, "Multiple M2-branes and the Embedding Tensor," arXiv:0804.2201 [hep-th]. K. Hosomichi, K. M. Lee and S. Lee, "Mass-Deformed Bagger-Lambert Theory and its BPS Objects," arXiv:0804.2519 [hep-th]. G. Papadopoulos, "On the structure of k-Lie algebras," arXiv: 0804.3567 [hep-th].

[20] P. Pasti, D. P. Sorokin and M. Tonin, "Covariant action for a D = 11 five-brane with the chiral 
field," Phys. Lett. B 398, 41 (1997) [arXiv:hep-th/9701037]; I. A. Bandos, K. Lechner, A. Nurmagambetov, P. Pasti, D. P. Sorokin and M. Tonin, "Covariant action for the super-five-brane of M-theory," Phys. Rev. Lett. 78, 4332 (1997) [arXiv:hep-th/9701149];

[21] M. Aganagic, J. Park, C. Popescu and J. H. Schwarz, "World-volume action of the M-theory five-brane," Nucl. Phys. B 496, 191 (1997) [arXiv:hep-th/9701166].

[22] A. Basu and J. A. Harvey, "The M2-M5 brane system and a generalized Nahm's equation," Nucl. Phys. B 713, 136 (2005) [arXiv:hep-th/0412310].

[23] B. de Wit, J. Hoppe and H. Nicolai, "On the quantum mechanics of supermembranes," Nucl. Phys. B 305, 545 (1988).

[24] T. Banks, W. Fischler, S. H. Shenker and L. Susskind, "M theory as a matrix model: A conjecture," Phys. Rev. D 55, 5112 (1997) [arXiv:hep-th/9610043].

[25] N. Ishibashi, H. Kawai, Y. Kitazawa and A. Tsuchiya, "A large-N reduced model as superstring," Nucl. Phys. B 498, 467 (1997) [arXiv:hep-th/9612115].

[26] C. S. Chu and P. M. Ho, "Noncommutative open string and D-brane," Nucl. Phys. B 550, 151 (1999) [arXiv:hep-th/9812219]. C. S. Chu and P. M. Ho, "Constrained quantization of open string in background B field and noncommutative D-brane," Nucl. Phys. B 568, 447 (2000) [arXiv:hep-th/9906192]. V. Schomerus, "D-branes and deformation quantization," JHEP 9906, 030 (1999) [arXiv:hep-th/9903205]. N. Seiberg and E. Witten, JHEP 9909, 032 (1999) [arXiv:hepth/9908142].

[27] P. M. Ho and Y. Matsuo, "A toy model of open membrane field theory in constant 3-form flux," Gen. Rel. Grav. 39, 913 (2007) [arXiv:hep-th/0701130]. 